\title{
Multiple Giant Coronary Artery Aneurysms
}

\author{
Hiroki Usuku ${ }^{1,2}$, Sunao Kojima ${ }^{1}$, Naoto Kuyama ${ }^{1}$, Shinsuke Hanatani ${ }^{1}$, Satoshi Araki ${ }^{1}$, \\ Kenichi Tsujita ${ }^{1}$, Ryusuke Tsunoda ${ }^{2}$, Toshihiro Fukui ${ }^{3}$ and Seiji Hokimoto ${ }^{1}$
}

\begin{abstract}
A 74-year-old man was admitted to our hospital with chest pain and dyspnea associated with ST elevation in leads II, III and aVF. An echocardiogram showed an enlarged mass lesion measuring nearly $80 \mathrm{~mm}$. Coronary angiography showed two giant coronary artery aneurysms (CAAs) in the right coronary artery (RCA). CAAs were also seen in the left main trunk and left anterior descending artery. Computed tomography showed the CAA in the RCA was ruptured into the right atrium. We therefore diagnosed this patient with multiple CAAs, myocardial infarction and coronary artery rupture. He underwent successful surgical excision and coronary bypass surgery.
\end{abstract}

Key words: giant coronary artery aneurysms, coronary artery rupture

(Intern Med 56: 1973-1976, 2017)

(DOI: 10.2169/internalmedicine.56.8357)

\section{Introduction}

A coronary artery aneurysm (CAA) is defined as a coronary dilatation that exceeds the diameter of the normal adjacent segments or with a diameter 1.5 times that of the patient's largest coronary vessel (1). The exact definition of giant CAA is still undetermined, although the term generally refers to a dilatation $>4$ times the diameter of a reference vessel or a vessel diameter exceeding $8 \mathrm{~mm}$ (2). CAAs are caused by Kawasaki disease (febrile illness with mucosal inflammation), coronary atherosclerosis, trauma (including percutaneous coronary intervention), autoimmune diseases (polyarteritis nodosa, systemic lupus erythematosus, scleroderma) and coronary artery dissection, among others (3). Myocardial infarction (MI) and CAA rupture are known to be major fatal complications of giant CAAs. We successfully treated a case of multiple giant CAAs presenting with acute MI, CAA rupture and severe heart failure and report the details of this case.

\section{Case Report}

A 74-year-old man who presented with acute onset of central chest pain and severe dyspnea was admitted to our hospital. He had a history of hypertension and dyslipidemia but not Kawasaki disease or other vasculitises. On a physical examination, his blood pressure was 91/64 $\mathrm{mmHg}$, pulse rate was $88 \mathrm{bpm}$ and oxyhemoglobin saturation measured by pulse oximetry was $90 \%$ under $6 \mathrm{~L}$ of oxygen. No cardiac murmur was audible. Chest X-ray film showed an expanded heart shadow and pulmonary congestion (Fig. 1A). An electrocardiogram showed ST elevation in leads II, III and aVF (Fig. 1B). An ultrasonic echocardiogram showed a decreased left ventricular ejection fraction $(25 \%$ calculated by the modified Simpson method), moderate mitral regulation and an enlarged mass lesion measuring nearly $80 \mathrm{~mm}$ (Fig. 1C-E, white arrow). Emergent coronary angiography showed two giant coronary artery aneurysms (CAAs; Fig. 2A, white and red arrows) in the right coronary artery (RCA), which was totally occluded at the distal portions of the CAAs. There was severe coronary stenosis $(>90 \%)$ in the left main trunk (LMT), and the left circumflex artery was totally occluded (Fig. 2B, white arrows). CAAs were also seen in the LMT and the proximal portion of the left anterior descending artery (Fig. 2B, white rectangle). Emergent computed tomography showed an $85-\mathrm{mm}$ round mass in the RCA, which had ruptured into the right atrium

\footnotetext{
${ }^{1}$ Department of Cardiovascular Medicine, Kumamoto University Graduate School of Medical Sciences, Japan, ${ }^{2}$ Department of Cardiovascular Medicine, Japanese Red Cross Kumamoto Hospital, Japan and ${ }^{3}$ Department of Cardiovascular Surgery, Kumamoto University Graduate School of Medical Sciences, Japan

Received for publication October 5, 2016; Accepted for publication November 14, 2016

Correspondence to Dr. Sunao Kojima, kojimas@kumamoto-u.ac.jp
} 

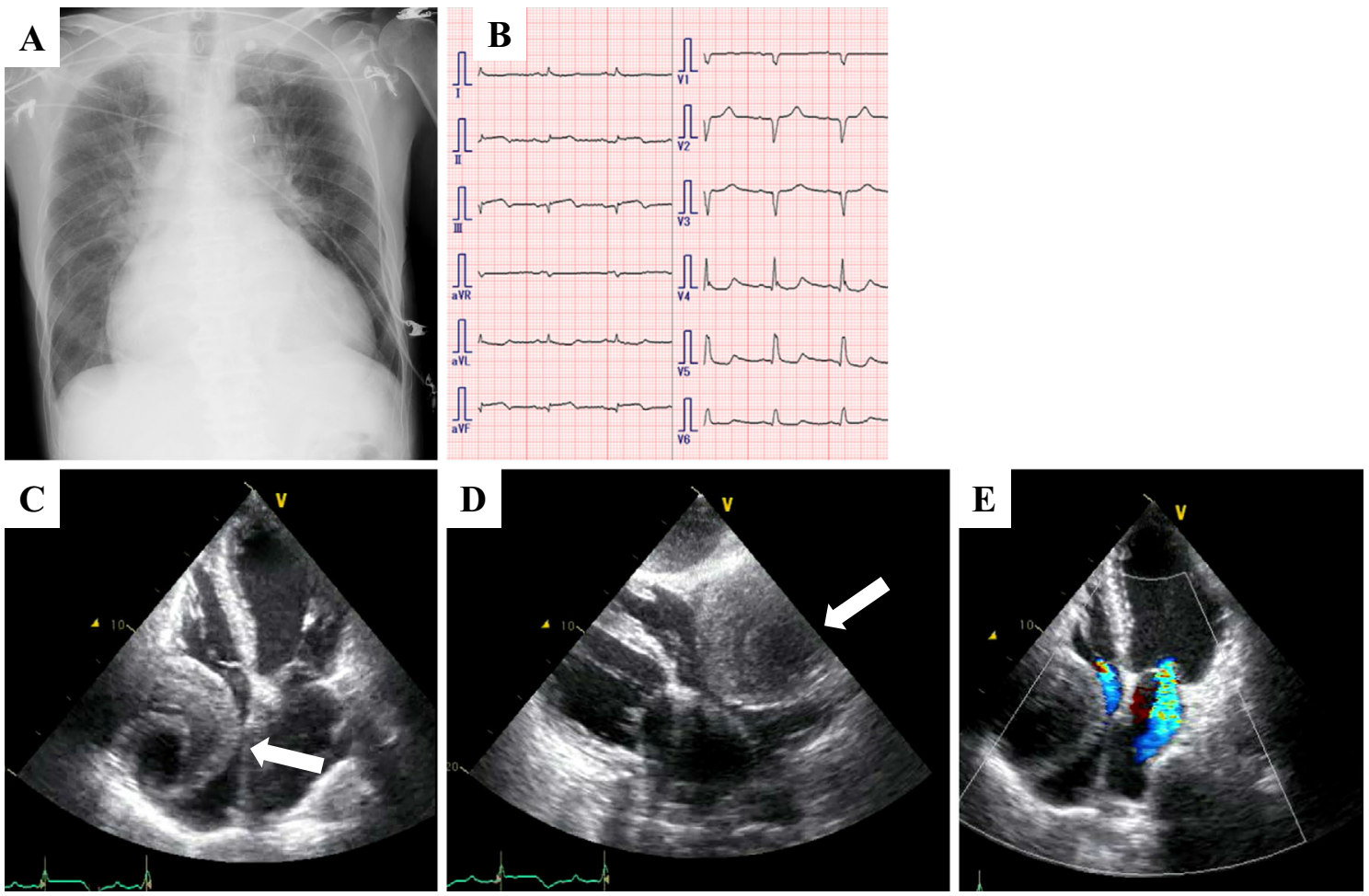

Figure 1. Chest X-ray film (A), electrocardiogram (B) and echocardiogram (C-E) of the patient early during admission at our hospital. An echocardiogram showed an enlarged mass lesion measuring nearly $80 \mathrm{~mm}$ (white arrow).
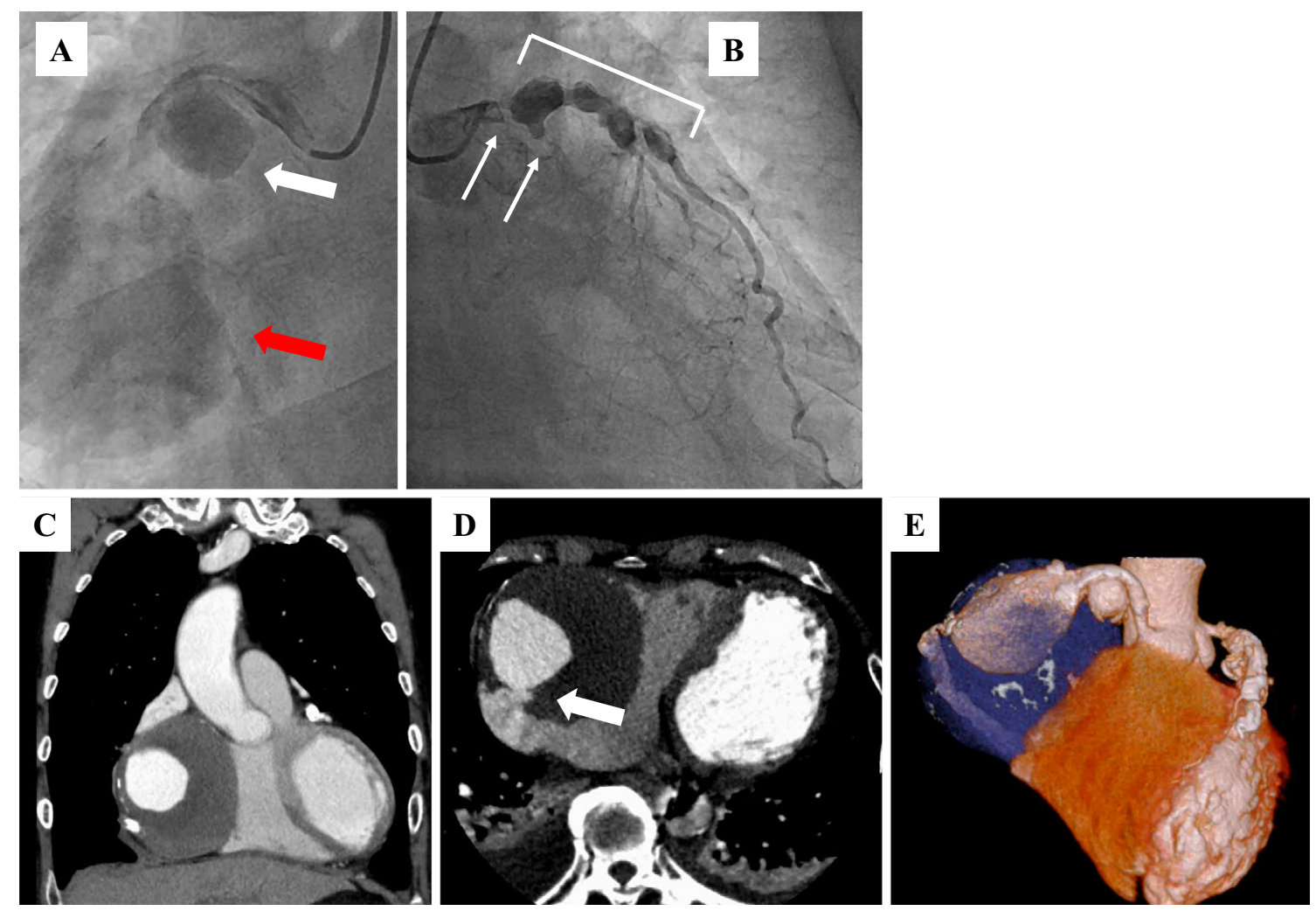

Figure 2. Emergent coronary angiography showed two giant coronary artery aneurysms (CAAs; A: white and red arrows) in the RCA, severe coronary stenosis (>90\%) in the left main trunk (LMT), an occluded left circumflex artery (B: white arrows) and multiple CAAs in the LMT and the proximal portion of the left anterior descending artery (B: white rectangle). Emergent computed tomography showed an 85-mm round mass in the RCA (C-E), which had ruptured into the right atrium (D: white arrow). 


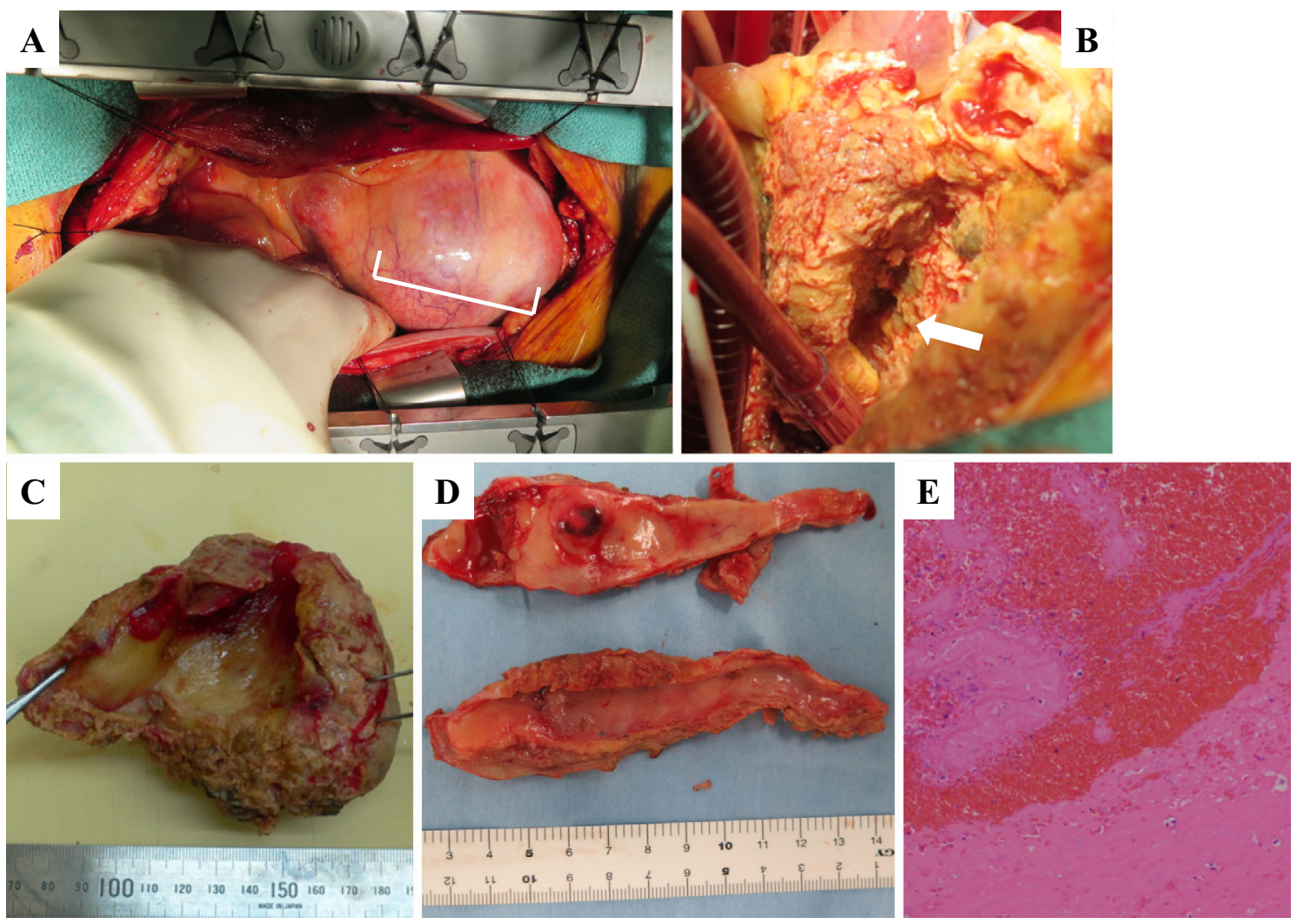

Figure 3. Intraoperative view of giant coronary artery aneurysm (A: white rectangle). After the giant CAA was removed. we could see the fistula from the coronary artery to the right atrium (B: white arrow). The resected aneurysm was filled with giant organized thrombi and fresh red thrombi (C). We observed severe atherosclerosis in the efferent vessels of the giant CAA (D). A pathological examination revealed organized thrombi and blood cells with no blood vessel components $(E)$.

(Fig. 2C-E, white arrow). We therefore diagnosed this patient with multiple CAAs, ST elevation MI and coronary artery rupture.The patient underwent emergent surgery (coronary artery bypass grafting, resection of CAAs and mitral annuloplasty). After median sternotomy, a giant CAA was identified (Fig. 3A, white rectangle). An aortic cannula was inserted into the ascending aorta, and two venous cannulae were inserted into the superior and inferior vena cava via the right atrium. After cardiopulmonary bypass was initiated, the aorta was cross-clamped. When the RCA aneurysm was removed, we could see a fistula entering the right atrium from the back of the CAA (Fig. 3B, white arrow). The proximal RCA was ligated at the ostium of the aneurysm, the distal RCA was ligated at the ostia, and the fistula to the right atrium was also ligated. We then dissected the right atrium and reached the left atrium through the atrial septum. We performed mitral annuloplasty using a physio II 28-mm ring (Edwards Lifesciences, Irvine, CA, USA). The left internal thoracic artery (LITA) was anastomosed to the distal $\mathrm{LAD}$, a saphenous vein graft (SVG) was anastomosed to the distal LCX, and another SVG was anastomosed to the distal RCA. The proximal ends of these two SVG were anastomosed to the ascending aorta.

The resected aneurysm was filled with giant organized thrombi and fresh red thrombi (Fig. 3C). Severe atherosclerosis was noted in the efferent vessels of the giant CAA
(Fig. 3D). A pathological examination revealed only organized thrombi and blood cells with no blood vessel components (Fig. 3E). Although acute heart failure occurred during the postoperative course, medical treatment improved this patient's condition, and he was transferred to a chronic care hospital for rehabilitation on the 34 th postoperative day.

\section{Discussion}

The estimated incidence of CAA is $0.3-5 \%$ among patients undergoing coronary artery angiography (1). In adults, giant CAA is an extremely rare entity with an estimated incidence of $0.02 \%$ in atherosclerotic cases (2). They occur in men more frequently than women. The most frequently affected coronary artery is the RCA. The causes of CAA are Kawasaki disease, coronary atherosclerosis, trauma, autoimmune diseases and coronary artery dissection, among others (3). Kawasaki disease is the most common cause of giant CAAs in Japan (4). It is a generalized vasculitis of unknown etiology and occurs in children. Coronary artery abnormalities develop in approximately $20 \%$ of children with untreated Kawasaki disease. Abnormalities of coronary arteries include ectasia or aneurysms that may be fusiform or saccular $(5,6)$. However, the present patient had no history of Kawasaki disease or a sustained high fever. In addition, no evidence of active vasculitis was observed in this pa- 
tient's aneurysm. Aside from Kawasaki disease, trauma and autoimmune diseases have also been causes of CAAs. However, our patient had no history of coronary intervention or autoimmune diseases. In adults, the most common cause of giant CAAs is atherosclerosis, accounting for $50 \%$ of reported cases (1). Notably, this patient had many coronary disease risk factors, including advanced age, hypertension, and dyslipidemia. Furthermore, severe atherosclerosis was seen in this patient's coronary arterial wall, and the CAAs were located on the distal portion of the coronary stenosis. When associated with coronary atherosclerosis, CAAs are known to occur in areas of turbulent flow, leading to poststenotic dilation (7). We therefore suspect that atherosclerosis might have been the main cause of giant CAA in this case.

This was a very rare case of giant CAAs in which the patient survived despite CAA rupture and an ST segment elevation MI complicated by severe heart failure. MI and CAA rupture are major fatal complications for giant CAAs. It is difficult to explain the association between MI and CAA rupture. Bauer et al. speculated that thrombi, which form in CAAs, occupy the efferent artery and increase the intraluminal pressure, causing rupture of the weakened wall of the CAA (8). Contrarily, Salehi et al. speculated that the altered blood flow or hemostasis resulting from rupture of the CAA might cause MI (9). In the present case, we observed fresh red thrombi and lots of organized thrombi in the resected CAA. We therefore suspect that occlusion of the outflow of the coronary aneurysm by thrombi might have directly caused the CAA rupture.

Management of CAA varies according to the etiology, size and symptoms. Generally, small, asymptomatic aneurysms without coronary artery disease are managed with conservative treatment. Warfarin anticoagulation therapy has been shown to be highly effective at reducing the risk of myocardial infarction or sudden cardiac death in patients with coronary aneurysm (10). Giant CAA with any symptoms are usually managed with surgical exclusion via resection or ligation (11). Closure of the fistula is also mandatory if giant CAA are combined with a fistula (12).

In conclusion, an MI patient with heart failure and ruptured giant CAAs with perforation into the right atrium was successfully treated with emergent surgical repair and inten- sive medical management.

The authors state that they have no Conflict of Interest (COI).

\section{Acknowledgement}

The authors thank Shinji Sumiyoshi from the Department of Pathology at Kumamoto University for his contributions.

\section{References}

1. Nichols L, Lagana S, Parwani A. Coronary artery aneurysm: a review and hypothesis regarding etiology. Arch Pathol Lab Med 132: 823-828, 2008.

2. Kato H, Sugimura T, Akagi T, et al. Long-term consequences of Kawasaki disease. A 10- to 21-year follow-up study of 594 patients. Circulation 94: 1379-1385, 1996.

3. Wright WP, Alpert MA, Mukerji V, Santolin CJ. Coronary artery aneurysms: a case study and literature review. Angiology 42: 672679, 1991.

4. Nakamura Y, Yashiro M, Uehara R, et al. Case-control study of giant coronary aneurysms due to Kawasaki disease. Pediatr Int 45: 410-413, 2003.

5. Dajani AS, Taubert KA, Gerber MA, et al. Diagnosis and therapy of Kawasaki disease in children. Circulation 87: 1776-1780, 1993.

6. Gersony WM. Diagnosis and management of Kawasaki disease. JAMA 265: 2699-2703, 1991.

7. Daoud AS, Pankin D, Tulgan H, Florentin RA. Aneurysms of the coronary artery. Report of ten cases and review of literature. Am J Cardiol 11: 228-237, 1963.

8. Bauer HH, Allmendinger PD, Flaherty J, Owlia D, Rossi MA, Chen C. Congenital coronary arteriovenous fistula: spontaneous rupture and cardiac tamponade. Ann Thorac Surg 62: 1521-1523, 1996.

9. Salehi N, Samiei N, Pouraliakbar H, Sabet AH, Vakili-Zarch A. Giant aneurysmal fistula of the left main coronary artery to the right atrium. Heart Surg Forum 15: E292-E293.

10. Sugahara $Y$, Ishii $M$, Muta $H$, Iemura $M$, Matsuishi $T$, Kato $H$. Warfarin therapy for giant aneurysm prevents myocardial infarction in Kawasaki disease. Pediatr Cardiol 29: 398-401, 2008.

11. Li D, Wu Q, Sun L, et al. Surgical treatment of giant coronary artery aneurysm. J Thorac Cardiovasc Surg 130: 817-821, 2005.

12. Bobos D, Chatzis AC, Giannopoulos NM, et al. Successful surgical repair of a giant arteriovenous fistula of the coronary arteries. J Card Surg 21: 269-270, 2006.

The Internal Medicine is an Open Access article distributed under the Creative Commons Attribution-NonCommercial-NoDerivatives 4.0 International License. To view the details of this license, please visit (https://creativecommons.org/licenses/ by-nc-nd/4.0/).

(C) 2017 The Japanese Society of Internal Medicine http://www.naika.or.jp/imonline/index.html 